\title{
Sobrepeso, obesidad, niveles de actividad física y autoestima de la niñez centroamericana: un análisis comparativo entre países \\ Overweight, obesity, physical activity levels, and self-esteem in Central American children: comparative analysis between countries
}

Carlos Eduardo Alvarez, María Fernanda Herrera Monge, Emmanuel Herrera González, Grettel Villalobos Víquez, Gerardo

Araya Vargas

Universidad Nacional de Costa Rica

\begin{abstract}
Resumen. El propósito de esta investigación fue examinar la prevalencia de sobrepeso, obesidad, niveles de actividad física y de autoestima de la población infantil centroamericana, comparándoles según sexo y país. Este estudio comparativo transversal de muestra probabilística aleatoria por conglomerados evaluó 5291 estudiantes matriculados en cuarto, quinto y sexto grado, de los cuales $52.3 \%$ fueron niñas y 47.7\% niños, pertenecientes a 73 centros educativos de Guatemala, Honduras, El Salvador, Costa Rica, Nicaragua y Panamá, con una edad promedio de $10.90 \pm 1$ 13. En todos los países se realizó la medición del peso y la talla para el cálculo del índice de masa corporal, se aplicó el cuestionario de actividad física para niños y niñas mayores (PAQ-C) y el cuestionario LAWSEQ para la medición del nivel de autoestima. Los resultados de esta investigación mostraron una prevalencia de sobrepeso y obesidad del 25\%, un nivel de actividad física bajo en un 35.1\% y una baja autoestima en el $44.3 \%$. Asimismo, al determinar el efecto del país de procedencia y del género sobre el índice de masa corporal, nivel de actividad física y autoestima, controlando la edad como una covariable, se encontró que El Salvador presentó el mayor promedio de índice de masa corporal y el menor nivel de actividad física junto a Honduras y Panamá. En relación a la autoestima, solamente Costa Rica y Guatemala presentaron una autoestima promedio. Esto demuestra que la situación de salud pública que enfrenta la niñez centroamericana es un problema que debe ser atendido en forma integral.
\end{abstract}

Palabras claves: estado de salud, índice de masa corporal, promoción de la salud escolar, autopercepción, comportamiento infantil.

Abstract. The purpose of this investigation was to explore the prevalence of overweight, obesity, physical activity levels, and selfesteem in Central American children, comparing them by gender and country. This comparative cross-sectional study used a random probabilistic conglomerate-based sampling technique, by which 5.291 students ( $52.3 \%$ girls and $47.7 \%$ boys) enrolled in fourth, fifth, and sixth grade from 73 schools in Guatemala, Honduras, El Salvador, Costa Rica, Nicaragua, and Panama, with an average age of 10.90 \pm 1.13 years old, were selected. In all the participating countries, weight and height were taken to calculate body mass index; additionally, the Physical Activity Questionnaire for Children (PAQ-C) and the Lawrence's Self-Esteem Questionnaire (LAWSEQ) were applied. The results suggest a prevalence of overweight and obesity (25\%), low physical activity levels (35.1\%), and low self-esteem (44.3\%). Also, when analyzing variables interaction by country and gender, it was found that El Salvador presented the highest average body mass index and the lowest levels of physical activity, along with Honduras and Panama. Regarding self-esteem, only Costa Rica and Guatemala presented average rank. This shows that the conditions of public health with regard to Central American youth are concerning and must be dealt with in an integral manner.

Key words: health status, body mass index, school health promotion, self-perception, Child behavior.

\section{Introducción}

La niñez enfrenta diferentes situaciones que influyen en su desarrollo, pero desde finales del siglo XX y durante las dos primeras décadas del siglo XXI, han ganado importancia las problemáticas por inactividad física, el sobrepeso, la obesidad y factores de orden psicológico, como elementos de riesgo que atentan contra la salud de esta población, así como para otros grupos de edad, y según varias evidencias, estos problemas tienen orígenes multifactoriales, tanto sociales, fisiológicos, metabólicos, moleculares, como genéticos (Cigarroa, Sarqui \& Zapata-Lamana, 2016; García, Calahorro, Torres \& Larra, 2010).

Particularmente, el sobrepeso y la obesidad en la niñez se han destacado a nivel mundial como uno de los principales factores de riesgo para el desarrollo de las enfermedades crónicas no transmisibles [ECNT], tales como enfermedades cardiacas, diabetes mellitus, hipertensión arterial y ciertos tipos de cáncer (Consejo de Ministros de Salud de Centroamérica y República. Dominicana [COMISCA], 2013). Además del peso corporal excesivo, la mala alimentación y la

\footnotetext{
Fecha recepción: 22-03-19. Fecha de aceptación: 21-08-19 Carlos Eduardo Alvarez
} ceab.03@gmail.com falta de actividad física (a su vez fuertemente relacionados con el sobrepeso y la obesidad) son factores que favorecen esta epidemia mundial (Savino, 2011). Pero además, el peso excesivo en infantes se ha asociado con trastornos psicosociales, en especial con problemas en autoestima, así como con un bajo desempeño físico en esta población (ver por ejemplo, los resultados del estudio de Delgado-Floody, Caamaño-Navarrete, Jerez-Mayorga et al, 2017, realizado en escolares chilenos). Por tanto, es necesario examinar, además de la problemática de sobrepeso y obesidad, los hábitos de actividad física y variables psicosociales relacionadas, en esta población.

Según la Organización Mundial de la Salud [OMS] (2000), el sobrepeso y la obesidad han sobrepasado la prevalencia de la desnutrición, por tal motivo se han realizado estudios en los diferentes países de la región centroamericana, como insumo para la promoción de diferentes estrategias que mejoren los hábitos de vida de la niñez. Además, diferentes evidencias científicas han demostrado que entre más temprano se presenten estas afectaciones, mayor es el riesgo de padecer enfermedades o problemas como los ya señalados, de tener una muerte prematura y de reducir la esperanza de vida, lo cual justifica su estudio en niños, niñas y adolescentes (COMISCA, 2013; OMS y OPS, 2014). 
En todos los países centroamericanos se ha observado un aumento en los índices de sobrepeso y obesidad (aspecto que se ha estudiado más detenidamente en la población infantil de la región), así como la disminución de los niveles de actividad física (fenómeno poco estudiado a nivel escolar, pese a su importancia), lo cual puede afectar directamente los componentes psicosociales como la autoestima y la aceptación social en la población infantil, según distintas evidencias (Fernández, 2014; Macias, Gordillo y Camacho, 2012; Medina, Yuja y Lanza, 2016).

Antecedentes de la región sobre la prevalencia de sobrepeso y obesidad indican que, en Guatemala, para el 2015 se estimaba que uno de cada 20 infantes presentaba obesidad (Diéguez, 2015). En el Salvador, los más recientes datos oficiales del MINSAL (2016), indicaron una prevalencia del 23\% en niños entre los 7 a 9 años de edad. En Honduras, Medina et al (2016) reportaron para tres escuelas del país, un $27.4 \%$ de sujetos con esta condición. En Costa Rica, el censo de peso y talla realizado por el Ministerio de Salud y el Ministerio de Educación Pública (2016a) mostró un índice de sobrepeso y obesidad del 34\% en la población escolar de 6 a 12 años. Asimismo, en Nicaragua análisis realizados en la Encuesta Nicaragüense de Demografía y Salud [ENDESA] (2012), citada por INIDE (2013) reportaron que los niños en edades de 4 a 17 años presentaron un índice de obesidad de $30 \%$. Y en el caso de Panamá, para el 2008, se reportó una prevalencia del 25.4\%, según el MEF (2008).

En el caso de la actividad física Klein (2008), señala que su oferta a nivel mundial ha disminuido con el tiempo. Para Hernández, Ferrando, Quílez, Argonés y Terreros (2010), uno de los principales factores del sedentarismo, es la reducción del tiempo dedicado a educación física, en los centros educativos, y su asignación a materias meramente teóricas, lo que ocasionará a largo plazo que la niñez y juventud no sientan la necesidad de tener a la actividad física como un referente de un hábito de vida saludable.

Lastimosamente, en Centroamérica la falta de evidencia científica en este tema ha generado un desconocimiento de las consecuencias del sedentarismo en la niñez. En países como Guatemala, los datos de la Dirección General de Educación Física [DIGEF] citado por Muñoz (2017), mencionan que en el país solamente tres de cada 10 estudiantes reciben clases de educación física dentro del centro educativo. Sin embargo, Muñoz (2017) señala que únicamente el 18\% de los niños cuentan con un profesional especializado en la enseñanza de la educación física en este país. En Costa Rica, el Departamento de Análisis Estadístico del Ministerio de Educación Pública, citado por Alfaro (2016) señaló que, aproximadamente un $30 \%$ de la población estudiantil no recibe educación física en la escuela y que el $70 \%$ que sí cursan esta materia, apenas tienen 80 minutos semanales para realizarla, y este tiempo se reduce a 50 minutos o menos por elementos meramente administrativos, como lo menciona Álvarez (2018) en el estudio sobre el análisis de los niveles de actividad física de los escolares, el tipo de contenido de la lección y la interacción del profesor durante la clase de educación física de instituciones públicas. Asimismo, en Panamá se ha reducido drásticamente las horas dedicadas a la práctica de actividad física en las escuelas y los colegios (Espinosa, 2013).
Aunado a los problemas en el peso corporal y los estilos de vida inactivos, los componentes psicosociales tienen una relación directa en la población infantil, específicamente en factores como la aceptación social y la autoestima (ContrerasValdez, Hernández-Guzmán \& Freyre, 2016). La baja autoestima en los infantes es cada vez más común, en una sociedad donde la identidad personal depende directamente de los estereotipos que dicta la sociedad (Delgado-Floody, Caamaño, Osorio et al., 2017; Sánchez y Ruíz, 2015). Por lo que, la población infantil que presenta esta problemática tiene más probabilidades de presentar problemas en la autopercepción y por ende verse afectados en aspectos como la interacción social y el rendimiento académico en general (Zanatta, Fuentes, van Barneveld, Medina \& Escobar, 2014).

Sin embargo, si se comparan estos datos con los resultados obtenidos en recientes investigaciones, realizadas con esta visión integral de la salud se puede observar la alarmante situación que vive actualmente la población infantil centroamericana. En el caso de Guatemala, la investigación realizada por Dubón \& Aguilar (2018) en centros educativos del centro de la capital, mostró que cuatro de cada 10 escolares entre los 9 y los 13 años presentaron altos índices de sobrepeso y obesidad, el $18.5 \%$ se percibió con un bajo nivel de actividad física y un $31.7 \%$ con baja autoestima. En el Salvador, se encontró una prevalencia del $23.3 \%$ de sobrepeso en los niños y niñas de 9 a 13 años, en 11 escuelas de la capital del país, un bajo nivel de actividad física en un 53.7\% y una baja autoestima en el 52.9\% (Evert \& Álvarez, 2018). Seguido de Honduras que si bien presentó los índices más bajos de sobrepeso y obesidad de la región, con una prevalencia del 8\% en Tegucigalpa y del 14.7\% en San Pedro Sula, se encuentra viviendo un fenómeno preocupante para la salud al presentar «la doble carga de la malnutrición» en donde los cambios en los estilos de vida de la población en general, ha ocasionado un incremento significativo en los niveles de sobrepeso y obesidad aun cuando otros segmentos de la población todavía presentan problemas de desnutrición muy marcados (Instituto de Nutrición de Centroamérica y Panamá [INCAP], 2015). En cuanto a la actividad física, un $47 \%$ y $26.4 \%$ respectivamente, presentaron bajos niveles y en relación a la autoestima el 52.4\% de niños en Tegucigalpa y el 46.9\% en San Pedro Sula presentaron baja autopercepción (Lacayo, Check, Vega \& Córdova, 2018; Vásquez-Bonilla, Zelaya-Paz \& García-Aguilar, 2018).

Por otro lado, en Costa Rica, en un estudio reciente (Herrera-Monge et al., 2019) con población escolar, un 34.2\% de los estudiantes evaluados presentaron sobrepeso y obesidad, un 29.4\% indicó percibirse con bajo nivel de actividad física y un $26.9 \%$ con baja autoestima. En el caso de Nicaragua, se encontró una prevalencia de $25 \%$ en 731 estudiantes de edad escolar, un $18 \%$ con sedentarismo y un $51 \%$ con problemas de baja percepción de sí mismos (Reyes, Otero, Pastrán, Herrera-Monge \& Álvarez, 2018). Por último, Panamá con un 29.7\% de estudiantes con problemas de exceso en el peso corporal, un $43.5 \%$ con baja actividad física y $43.9 \%$ con baja autoestima (Ambulo, González \& Montenegro, 2018).

Ante estos resultados, queda claro que los esfuerzos realizados en la región continúan siendo escasos y aislados, de ahí el incremento de esta problemática en salud. Es por tal motivo que el propósito de esta investigación fue examinar 
la prevalencia de sobrepeso, obesidad, niveles de actividad física y de autoestima de la población infantil centroamericana, comparándoles según sexo y país, con el fin de colaborar en el replanteamiento de estrategias y acciones que contribuyan en la disminución de esta problemática a nivel escolar en la región.

\section{Método}

\section{Participantes}

Este estudio fue de tipo comparativo transversal de muestra probabilística aleatoria por conglomerados (HernándezSampieri, Fernández-Collado \& Baptista, 2010). Se pretendía tener muestras representativas de la población escolar de los países adscritos a la Red Centroamericana de Investigación Aplicada en Niñez y Adolescencia en Movimiento Humano (REDCIMOVI) pero por razones diversas (problemas de seguridad, falta de recurso económico, etc.) se delimitó la población a estudiar, a escolares de cuarto al sexto grado, cuya escuela se localizara en el circuito, zona, distrito o departamento más cercano a la sede universitaria de cada país, que realizaría las evaluaciones. Este fue el universo de estudio y los centros educativos fueron los conglomerados. Mediante fórmula (Hernández-Sampieri et al, 2010) se estableció la muestra mínima de escuelas, y en cada una, se eligió un grupo de cada grado (cuarto a sexto), para evaluar a todos sus estudiantes. Por tanto, las muestras examinadas representan a la población escolar de una zona específica de cada país.

Se evaluaron 5291 estudiantes matriculados en cuarto $33 \%(n=1744)$, quinto $34.2 \%(n=1811)$ y sexto grado $32.8 \%$ ( $n=1736)$, de los cuales 52.3\% ( $n=2767)$ fueron niñas y $47.7 \%$ $(n=2524)$ fueron niños pertenecientes a 73 centros educativos de Guatemala (10 escuelas, 406 niños, 558 niñas), Honduras (20 escuelas, 766 niños, 878 niñas), El Salvador (11 escuelas, 399 niños, 359 niñas), Costa Rica (10 escuelas, 234 niños, 208 niñas), Nicaragua (9 escuelas, 366 niños, 365 niñas) y Panamá (13 escuelas, 353 niños, 399 niñas), con edad promedio de $10.90 \pm 1.13$ años.

Además, para la selección de las escuelas participantes se tomó en cuenta que al menos estas contaran con 100 estudiantes matriculados en el ciclo lectivo en los grados de cuarto, quinto y sexto, que los participantes tuvieran una edad entre los 9 y 13 años y que se tuviera la aprobación de las autoridades para aplicar las mediciones. De no cumplirse con alguno de los criterios de inclusión mencionados o de presentar los sujetos, al momento de las mediciones, alguna lesión o enfermedad que pudiera alterar sus resultados, debían ser excluidos.

\section{Instrumentos}

A continuación, se describe cada uno de los instrumentos que se utilizaron:

\section{Índice de Masa Corporal}

Para establecer el índice de masa corporal (IMC) se midió la talla y el peso de cada participante. La talla, con el estadiómetro móvil, marca seca 213. Para ello se le solicitó al niño colocarse de espalda contra el estadiómetro, seguido de tomar y anotar la medida. El peso fue medido a través de una balanza modelo Tanita UM-081, solicitando al niño subirse a la balanza sin calzado y registrando el dato en kilogramos, siguiendo el protocolo establecido por el Ministerio de Salud Pública y el Ministerio de Educación Pública (2016b) en el censo escolar de peso/talla. Para calcular el índice de masa corporal de cada estudiante se utilizó la calculadora para la población infantil y juvenil establecida por el Centro de Control y Prevención de Enfermedades [CDC] (2015) de los Estados Unidos en su sitio web, que estima el IMC por percentil de edad, según los cuadros de crecimiento por edad, a partir de la fecha de nacimiento, el día de la medición, el peso y la talla.

\section{Nivel de Actividad Física}

Cuestionario de Actividad Física para Niños y Niñas Mayores (PAQ-C), en inglés, «Physical Activity Questionnaire for Older Children», con siglas PAQ-C (Crocker, Bailey, Faulkner, Kowalski \& McGrath, 1997), validado al español por Martínez-Gómez et al. (2009). Es un instrumento indicado para niños y niñas con edades entre los 8 y 14 años, que proporciona una puntuación general de actividad física durante los últimos siete días, en diferentes situaciones y diferentes momentos del día. Cuenta con nueve preguntas cerradas de selección múltiple con escala de Likert que se puntúan de uno a cinco, siendo cinco una persona activa. El PAQ-C demuestra una adecuada confiabilidad test-retest $(r=$ 0.75 a 0.82 ) y una validez razonable ( $r=0.45$ a 0.53 ) (Crocker et al., 1997; Janz, Lutuchy, Wenthe \& Levy, 2008).

\section{Autoestima}

Cuestionario sobre Autoestima LAWSEQ, en inglés Lawrence Self-Esteem Questionnaire (LAWSEQ), adaptado al español por Marcó \& de García (2000), valora el nivel de autoestima en la población infantil, con base en la suma de características físicas y cognitivas de sí mismo. Cuenta con un total de 16 preguntas que valoran directamente la percepción sobre sí mismo. La fiabilidad interna de la escala se reporta $a ́=0.76$ (Lawrence, 1981) y tiene una aceptable validez externa $(r=0.73)$, con respecto al Coopersmith Self-Esteem Inventory.

\section{Procedimiento}

Se contactó a los diferentes Directores Regionales o Departamentales del Ministerio de Educación de cada país, para poder obtener la autorización para ejecutar las mediciones. Luego se solicitó en cada centro educativo participante la aprobación de los directores (as) explicándoles el objetivo de la investigación. Una vez obtenido el aval, se contactó a las maestras de cuarto, quinto y sexto grado para explicarles la importancia del estudio. Posteriormente, una vez obtenido el consentimiento informado por parte de encargados legales de los niños y el asentimiento de todos los niños participantes, en los países que así lo solicitaron, se recolectaron los datos respecto al grado escolar, el género y la edad, el peso, la talla, el índice de masa corporal, el nivel de actividad física y de autoestima, y para ello se estableció en cada país un calendario de visitas por escuela, para realizar las mediciones. Se realizó una única visita a cada escuela. En estas se procedió primeramente a aplicar los cuestionarios de actividad física y de autoestima (PAQ-C y LAWSEQ) y una vez 
completados los cuestionarios, se midió el peso y la talla de manera individual según los protocolos establecidos. Todas estas mediciones se realizaron en un día regular de clases y en presencia de la maestra o maestro de aula. Esta investigación se realizó bajo el estricto cumplimiento de los principios bioéticos establecidos por los diferentes entes y legislación de cada país.

\section{Análisis de datos}

Los datos se tabularon y fueron analizados por medio del paquete estadístico SPSS 24.0 para Windows. Se comprobó los supuestos de normalidad de los datos. Se realizó un análisis estadístico de tipo descriptivo (valores mínimos, máximos, medias y desviación estándar) para todas las variables de estudio IMC, PAQ-C y autoestima, en general y según sexo. Además de frecuencias absolutas y porcentajes para los casos de sobrepeso u obesidad (SOB), baja autoestima (BAU) y baja actividad física (BAF). Para examinar la relación entre las prevalencias de SOB, BAU y BAF, con respecto al sexo y al país, se realizaron análisis de Chicuadrado en tablas de contingencia de tres dimensiones. También se aplicó el análisis multivariado de covarianza (MANCOVA) para determinar el efecto del sexo y el país de procedencia sobre las variables dependientes (índice de masa corporal, nivel de actividad física y autoestima) controlando la edad como una covariable, dada su correlación estadísticamente significativa con esas variables (todas $p<0.001)$. Previamente, se comprobó el cumplimiento de los supuestos de la prueba (siguiendo lo indicado por O' Donoghue, 2012, y Meyers, Gamst \& Guarino, 2013). Se utilizó como criterio estadístico para prueba de hipótesis el nivel de significancia de $p<0.05$.

\section{Resultados}

Según los resultados de los análisis de Chi-cuadrado (ver tabla 1 y notas respectivas), tanto las mujeres como los

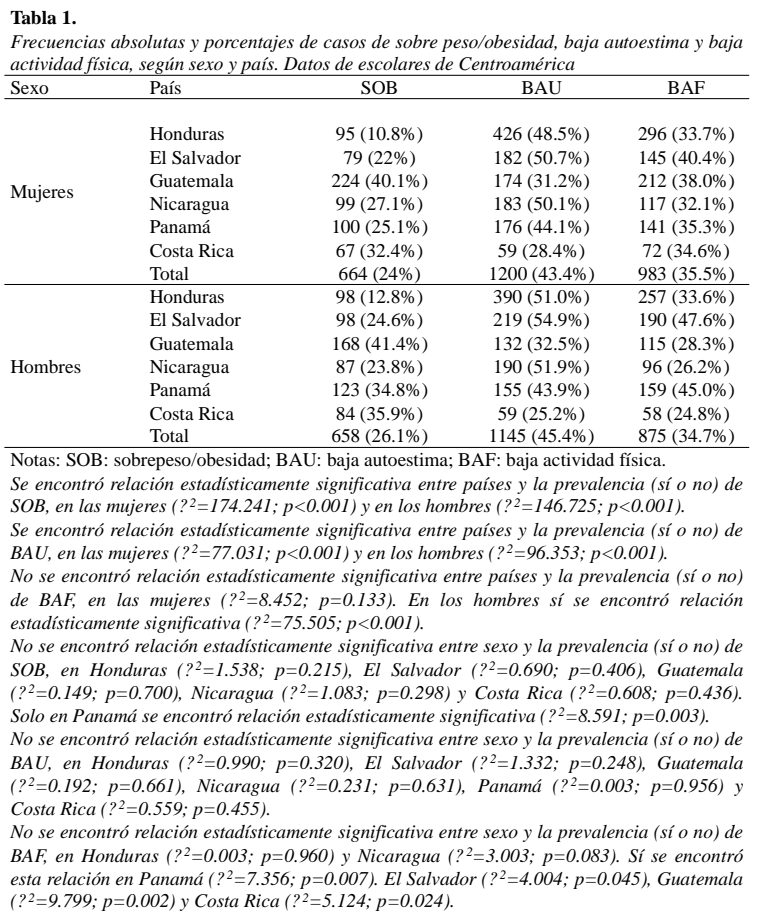

hombres escolares de Guatemala, tuvieron la mayor prevalencia de SOB (40.1\% y 41.4\% respectivamente). Y solo hubo diferencias entre las prevalencias de hombres y mujeres en Panamá (los hombres tuvieron mayor prevalencia de SOB que las mujeres: $34.8 \%$ v.s. $25.1 \%$ respectivamente).

En cuanto a la prevalencia de BAU, las mujeres de El Salvador (50.7\%) y Nicaragua (50.1\%), tuvieron las más altas, mientras que en los hombres, la prevalencia de BAU más alta se observó en El Salvador (54.9\%). Además, no se encontró diferencias en las prevalencias de BAU entre hombres y mujeres en ninguno de los países.

Finalmente, con respecto a la prevalencia de BAF, los hombres de El Salvador (47.6\%) y Panamá (45\%) tuvieron las más altas. En el caso de las mujeres, no hubo diferencias en sus prevalencias de BAF entre países. Por otro lado, al comparar las prevalencias de BAF entre hombres y mujeres en cada país, se encontró que los hombres de Panamá y El Salvador tuvieron mayor prevalencia de BAF que las mujeres de sus respectivos países (45\% v.s. $35.3 \%$ y $47.6 \%$ v.s. $40.4 \%$, respectivamente). Por el contrario, las mujeres de Guatemala y Costa Rica, tuvieron más prevalencia de BAF que los hombres de sus respectivos países (38\% v.s. 28.3\% y $34.6 \%$ v.s. $24.8 \%$, respectivamente).

En general, indistintamente del sexo o del país, se encontró que un $14.20 \%(n=749)$ de la muestra presentaba bajo peso para su edad, un $60.80 \%(n=3219)$ presentaba un peso saludable y un $24.9 \%$ ( $n=1322)$ tenía sobrepeso u obesidad. En cuanto al nivel actividad física, el 47.9\% (n=2535) de los estudiantes evaluados se percibían moderadamente activos, el 17\% ( $n=897)$ físicamente muy activos y el 35.1\% ( $\mathrm{n}=1858)$ con un bajo nivel de actividad física. Con respecto a la autoestima, los resultados demuestran que un $44.3 \%(n=2345)$ presentaba niveles bajos, un $48 \%(n=2540)$ reportó un nivel de autoestima promedio, y un 7.7\% (n=405) mostró una clasificación alta de autoestima.

Como resultados del MANCOVA de dos vías (sexo v.s. país) controlando la edad como covariable, que fue conducido para las variables dependientes índice de masa corporal, autoestima y actividad física, se encontró la prueba $\mathrm{M}$ de Box (de igualdad de las matrices de varianza-covarianza), estadísticamente significativa ( $M$ de Box=171.294; $p<0.001$ ), indicando así, que las matrices observadas de covarianza de las variables dependientes, fueron diferentes entre los niveles (grupos) de las variables independientes. Por tanto, se utilizó la traza de Pillai como estadístico para evaluar los efectos multivariados de este análisis. Vale mencionar que se encontró un resultado estadísticamente significativo en la prueba de esfericidad de Bartlett $\left(x^{2}=12486.522 ; p<0.001\right)$, indicando que existía suficiente correlación entre las variables dependientes, para proceder con este análisis multivariado.

Por otro lado, en la prueba de Levene de igualdad de varianzas de error, se encontró resultados estadísticamente significativos para las tres variables dependientes (todas con $p<0.001)$. Por tanto, para evaluar cualquier efecto univariado, de alguna de estas variables, se aplicó un nivel de significancia estadística más restrictivo $(p<0.001)$.

Mediante el examen de la traza de Pillai, se encontró que no hubo efecto multivariado estadísticamente significativo del sexo sobre las variables dependientes [Traza de Pillai=0, 
$F=0.441, p=0.724]$, pero sí existió efecto multivariado estadísticamente significativo del país [Traza de Pillai=0.91, $F=32.93, p<0.001]$ y de su interacción, es decir país v.s. sexo [Traza de Pillai $=0.99, F=4.74, p<0.001$ ]. Por tanto, el variato compuesto por el IMC, la autoestima y la actividad física reportada, tiende a ser diferente entre niños y niñas y entre países, asumiendo la edad como una constante.

Se realizó como seguimiento, ANOVA univariados para cada una de las variables dependientes por separado, para determinar el locus de los efectos multivariados observados. Además, se corrió el análisis post hoc de efectos simples, con el ajuste de comparaciones múltiples de Bonferroni, para examinar las interacciones univariadas respectivas. Según estos análisis, el efecto multivariado del país, se explica mediante efectos univariados del IMC $(F=28.390, p<0.001)$, la actividad física $(F=19.295, p<0.001)$ y la autoestima $(F=55.987, p<0.001)$, mientras que el efecto multivariado de la interacción entre país y sexo, solo se explica por el efecto univariado en actividad física ( $F=10.572, p<0.001)$.

La tabla 2 muestra los promedios ajustados y desviaciones estándar de las tres variables dependientes según el sexo y país de procedencia, controlando estadísticamente a la edad como una covariable. Específicamente, el post hoc muestra que los participantes evaluados de El Salvador $(p<0.001)$ difieren significativamente de los demás países, presentando el mayor promedio de índice de masa corporal. En los resultados de actividad física, El Salvador, Honduras y Panamá ( $\mathrm{p}=0.001$ ) presentan menor promedio de actividad física en comparación con Costa Rica, Nicaragua y Guatemala. En el caso de la variable autoestima, Guatemala, presenta diferencias significativas con todos los países, excepto con Costa Rica que presenta un nivel de autoestima promedioalto distinto a los demás países de la región centroamericana. Asimismo, el post hoc de la interacción país v.s. sexo en la variable actividad física, muestra que los hombres de Guatemala, Nicaragua y Costa Rica, tuvieron promedios mayores que los hombres de Honduras, El Salvador y Panamá. Mientras que en las mujeres no se observó diferencias en sus promedios de actividad física entre países. Finalmente, el post hoc de la interacción país v.s. sexo mostró que las mujeres tuvieron promedios mayores que los hombres en El Salvador y Panamá, y los hombres mostraron promedios mayores que las mujeres en Guatemala, Nicaragua y Costa Rica, mientras que en Honduras no se observó diferencias entre hombres y mujeres.

\begin{tabular}{|c|c|c|c|c|c|}
\hline Sexo & País & $n$ & IMC $\left(\mathrm{kg} / \mathrm{m}^{2}\right)$ & PAQ-C & Autoestima \\
\hline \multirow{6}{*}{ Niños } & Guatemala & 406 & $19.62 \pm 4.03$ & $3.28 \pm 0.78$ & $17.34 \pm 4.90$ \\
\hline & El Salvador & 399 & $21.50 \pm 4.04$ & $2.91 \pm 0.78$ & $14.05 \pm 4.92$ \\
\hline & Honduras & 766 & $19.57 \pm 4.03$ & $3.00 \pm 0.77$ & $15.29 \pm 4.92$ \\
\hline & Nicaragua & 366 & $18.75 \pm 4.03$ & $3.34 \pm 0.78$ & $14.90 \pm 4.91$ \\
\hline & Costa Rica & 234 & $19.56 \pm 4.02$ & $3.35 \pm 0.77$ & $18.15 \pm 4.87$ \\
\hline & Panamá & 353 & $19.73 \pm 4.03$ & $2.96 \pm 0.78$ & $15.83 \pm 4.91$ \\
\hline \multirow{6}{*}{ Niñas } & Guatemala & 558 & $19.96 \pm 4.02$ & $3.06 \pm 0.77$ & $17.31 \pm 4.90$ \\
\hline & El Salvador & 359 & $21.08 \pm 4.06$ & $3.03 \pm 0.78$ & $14.57 \pm 4.94$ \\
\hline & Honduras & 878 & $19.43 \pm 4.04$ & $3.06 \pm 0.77$ & $15.25 \pm 4.92$ \\
\hline & Nicaragua & 399 & $19.61 \pm 4.02$ & $3.20 \pm 0.78$ & $14.92 \pm 4.90$ \\
\hline & Costa Rica & 208 & $19.09 \pm 4.03$ & $3.18 \pm 0.78$ & $17.55 \pm 4.91$ \\
\hline & Panamá & 399 & $19.34 \pm 4.02$ & $3.18 \pm 0.78$ & $15.93 \pm 4.90$ \\
\hline
\end{tabular}

\section{Discusión}

El propósito de esta investigación fue comparar la pre- valencia de sobrepeso, obesidad, niveles de actividad física y de autoestima de la población infantil centroamericana con el fin de colaborar en el replanteamiento de estrategias y acciones que contribuyan en la disminución de esta problemática a nivel escolar en la región. Los hallazgos de este estudio indican que la niñez escolar centroamericana presenta una prevalencia de sobrepeso y obesidad de $24.9 \%$ y un $14.20 \%$ de bajo peso, los cuales dan evidencia del creciente problema de la mala nutrición como un fenómeno mundial, que afecta significativamente la salud de la población infantil (Catalani et al., 2016; González et al., 2017).

Al contrastar estos resultados con los de estudios de otras regiones del mundo, se aprecia que esta problemática detectada en la niñez centroamericana es incluso más grave que en algunas zonas. Por ejemplo, Mazidi et al (2018), realizaron una revisión sistemática y metaanálisis de estudios de prevalencia de sobrepeso y obesidad, realizados en niños y niñas (de 5 a 12 años de edad) y en adolescentes (de 12 a 19 años), de países de Asia, encontrando 5.8\% de obesidad (7\% en niños y $4.8 \%$ en niñas) y $11.2 \%$ de sobrepeso ( $11.7 \%$ en niños y $10.9 \%$ en niñas), siendo resultados inferiores a la prevalencia encontrada en el presente estudio.

En la región centroamericana esta situación es particularmente un problema de salud pública, ya que la mayor cantidad de reportes de sobrepeso y obesidad en la niñez desde 1990 indican un ascenso de esta problemática en comparación con otras regiones (Corvalán et al., 2017; Hruby \& Hu, 2015). Según el Instituto de Nutrición de Centroamérica y Panamá [INCAP] (2015), todos los países de la región presentan tasas de déficit y excesos en la alimentación y una alteración en los patrones de movimiento en la niñez, lo que evidencia la gran variabilidad que existe entre todos los países que la conforman; especialmente por las condiciones socioeconómicas que enfrentan cada uno. La rapidez con la que han crecido los países de ingresos medios y bajos, han generado un mayor auge en la prevalencia del sobrepeso y obesidad, ocasionando un incremento en el desarrollo de diferentes padecimientos a muy temprana edad, tales como las enfermedades crónicas no transmisibles [ECNT], deficiencias en el crecimiento de los niños, retraso en el desarrollo motor, problemas de desarrollo cognoscitivo e intelectual y trastornos psicosociales, los cuales pueden estar relacionados con problemas de discriminación social, generando conductas agresivas, de exclusión social y aislamiento, que a su vez contribuyen al desarrollo de ansiedad, depresión, baja autoestima, entre otros trastornos (Aceves-Martins, Llauradó, Tarro, Solà \& Giralt, 2016; Campo, 2014; Ceballos, Vizcarra, Acosta, Reyes \& Loza, 2012; Nestle, 2007; Solano, Baron \& Del Real, 2005) que, aunados a la desnutrición, implican un alto costo económico para los países debido a los gastos directos e indirectos que ocasionan ambas enfermedades (Shamah, 2016).

En el caso particular de esta región se presenta una situación denominada «la doble carga de la malnutrición», en los países de Guatemala, El Salvador, Honduras y Nicaragua, donde los hogares conviven diariamente con problemas de desnutrición crónica y altos niveles de sobrepeso y obesidad (INCAP, 2015), los cuales están relacionados a múltiples factores entre ellos: la mala alimentación, el sedentarismo y la historia familiar (Alba-Martin, 2016). Sumado a esta parti- 
cularidad, existe un incremento en la mayoría de países centroamericanos con respecto a la variable de sobrepeso y obesidad, principalmente en Guatemala, Costa Rica y Panamá, en los cuales evidentemente se ha generado un mayor desarrollo socioeconómico, que ha propiciado patrones alimenticios inadecuados y una adopción de estilos de vida inactivos, conducentes al desarrollo de estos problemas (Estado de la Región [ERCA], 2016).

Asimismo, cabe mencionar que pese a que este estudio no mostró diferencias estadísticamente significativas al integrar todos los países según sexo, existe una tendencia de presentar mayores índices de masa corporal en las niñas en comparación con los niños en países tales como Guatemala y Nicaragua, situación que ha sido reportada en abundantes investigaciones (Allen, Byrne, Blair \& Davis, 2006; Cambizaca Castañeda \& Sanabria, 2015; Martínez et al., 2017; Villagrán, Rodríguez-Martín, Novalbos, Martínez \& Lechuga, 2010), y que podría significar un acercamiento desde el punto de vista de educación diferenciado a la hora de intervenir a esta población. Por otro lado, al considerar el rango de edad evaluado en este estudio se afirma lo mencionado por Ceballos, et al. (2012) y Trejo, Jasso, Mollinedo \& Lugo (2012), quienes indican en su investigación que, los grados de cuarto a sexto grado escolar son periodos críticos en la determinación de condiciones y comportamientos que condicionarán la vida, por lo que si se presentan altos índices de sobrepeso y obesidad conforme se avanzan en estas edades hay una mayor posibilidad de convertirse en adultos con esta condición (Ministerio de Salud de la Nación, 2013; Liria, 2012).

Con relación a los niveles de actividad física, el 35.1\% presentó un nivel bajo y solamente un $17 \%$ se percibió con alto nivel; estos resultados al compararlos con los datos mencionados por INCAP (2016), quienes reportaron más de un $70 \%$ de estudiantes en edad escolar con bajos niveles de actividad física, según análisis de la región, indican una mejoría. No obstante, el bajo porcentaje de niños que reportaron realizar actividad física, cumpliendo las recomendaciones mundiales de la Organización Mundial de la Salud [OMS] (2010), demuestra la situación preocupante de sedentarismo en la niñez, sobre todo en países como El Salvador, Honduras y Panamá que tuvieron los resultados más altos de la región. Si bien, no existen evidencias de que el nivel de sedentarismo fue mayor en décadas pasadas, los patrones de movimiento de la niñez Centroamericana han cambiado, como resultado posiblemente de la reducción de espacios para jugar, el abuso de nuevas tecnologías, la disminución de oportunidades de actividad física en el entorno y los niveles de inseguridad, que han provocado un alto impacto en el porcentaje de niños con estilo de vida sedentario (Cofré, Muros \& Zurita, 2015; Lizardo \& Díaz, 2011; Miranda-Ríos et al., 2016).

Las conductas activas y sedentarias en la población infantil se presentan de manera muy heterogénea, debido a que ambos sexos dedican mucho tiempo a actividades sedentarias, principalmente conforme avanzan en la edad y se acercan a la adolescencia (Miranda-Ríos et al., 2016; Torres, Carpio, Lara \& Zagalaz, 2014). Sin embargo, existe una tendencia en las niñas a disminuir la práctica de actividad física a través del tiempo, a diferencia de los niños, quienes continúan teniendo el reforzamiento necesario para mantener un estilo de vida menos inactivo (De Piero, Rodríguez, González \& López, 2014; Raiman \& Verdugo, 2012).

Por tanto, es importante considerar las implicaciones del sedentarismo en la población infantil, ya que esto puede acarrear serios riesgos para la salud (OMS, 2018), además de que, acrecienta el riesgo de padecer sobrepeso u obesidad, duplica el riesgo de sufrir enfermedades cardiovasculares, hipertensión arterial, diabetes tipo II a temprana edad y disminuye la esperanza de vida de las personas (Delgado y Contreras, 2018). Igualmente, la falta de actividad física está asociada con menores rendimientos académicos y una deficiencia en el desarrollo de las habilidades psicomotoras en la niñez (Cigarroa, Sarqui \& Zapata-Lamana, 2016).

En el caso de la variable autoestima, un $44.3 \%$ de la niñez evaluada en general, presentó un nivel de autoestima bajo y solamente un 7,7\% un nivel de autoestima alto. Evidentemente la situación de niveles tan bajos de autoestima que enfrenta la niñez centroamericana es un problema de salud mental que debe ser atendido, especialmente en una sociedad en la que la identidad personal y la presión social son elementos que se les asigna una prioridad alta (Naranjo, 2007). De especial interés es enfatizar que, la situación de pobreza en esta área es una de las situaciones que influyen más ampliamente en el desarrollo de la autoestima de la niñez, ya que este constructo es en gran medida producto de la relación del infante con su medio y con quienes lo rodean, lo que provoca que, en condiciones de privación social, económica y cultural, constituya un factor de riesgo que influye sobremanera en la vulnerabilidad de la niñez centroamericana.

Según Sánchez \& Ruiz, (2015) y Taberno, Serrano \& Mérida, (2017), los ambientes de inseguridad social influyen a edades más tempranas en la construcción de una salud mental apropiada. Del mismo modo, se debe considerar que los niveles encontrados de baja autoestima en este estudio exponen a la población infantil evaluada a desordenes de alimentación y trastornos de la imagen corporal, lo que les impediría desarrollar una vida exitosa, causándoles deterioros en su bienestar psicológico (Zamani et al., 2016).

Una forma de lidiar con esta problemática es mediante la promoción de actividad física en escolares, dado que, científicamente, se ha reconocido que una de las mejores intervenciones desde la niñez, tomando en cuenta la influencia del ambiente, es la actividad física, para el desarrollo de una mejor salud física y mental, principalmente cuando esta se practica de moderada a vigorosa intensidad (Batista, Cubo, Honório \& Martins, 2016; Zamani et al., 2016). Al respecto, vale destacar la relación entre el sobrepeso y obesidad, y los niveles de sedentarismo de la población infantil, que se podría explicar como resultado de una disminución de la percepción de la autoeficacia y de la competencia física, lo que contrinuye a disminuir el nivel de práctica de actividad física, y esto a su vez, deteriora la aceptación social, lo que podría generar cambios evidentes en la autoestima en la niñez (Zamani et al., 2016) tal y como se ha mostrado en esta investigación.

\section{Conclusión}

La situación de salud pública que enfrenta la niñez cen- 
troamericana con respecto al sobrepeso, obesidad, sedentarismo y baja autoestima, es un problema que debe ser atendido en forma integral desde el ámbito educativo en la región centroamericana. Se detectó, a nivel centroamericano, una prevalencia de $25 \%$ en sobrepeso y obesidad, baja actividad física en el $35.1 \%$ y un $44.3 \%$ con baja autoestima, destacando diferencias entre países de la región, específicamente en Guatemala, Costa Rica y Panamá con niveles extremadamente altos de sobrepeso y obesidad en comparación con el resto, entre El Salvador, Honduras y Panamá con evidentes niveles de inactividad física y entre El Salvador, Honduras y Nicaragua, los cuales presentan los mayores niveles de baja autoestima. Esto refuerza la necesidad de enfatizar intervenciones en la edad escolar, ya que ha sido mostrado que es en la niñez donde se debe fortalecer el sistema de promoción de la salud y prevención de enfermedades, sobre todo porque es en esta etapa de vida donde se construyen las conductas apropiadas de salud.

\section{Agradecimientos}

Esta investigación fue financiada por la asociación ILSI Mesoamérica que forma parte de la red de entidades vinculadas al International Life Sciences Institute.

\section{Referencias}

Aceves-Martins, M., Llauradó, E., Tarro, L., Solà, R., \& Giralt, M. (2016). Obesity-promoting factors in Mexican children and adolescents: Challenges and opportunities. Global Health Action, 9(1). doi: https://doi.org/10.3402/gha.v9.29625

Alba-Martín, R. (2016). Prevalencia de obesidad infantil y hábitos alimentarios en educación primaria. Enfermería Global, 15(2), 40. doi: https://doi.org/10.6018/eglobal.15.2.212531

Alfaro, J. P. (2016). Más de 117.000 niños no reciben educación física en la escuela. Costa Rica: Periódico La Nación Digital. Recuperado de https://www.nacion.com/puro-deporte/otrosdeportes/mas-de-117-000-ninos-no-reciben-educacion-fisicaen-la-escuela/T6SLA6GMBBBYJMRQCMSZCZOFMY/ story/

Allen, K.L., Byrne, S.M., Blair, E.M., \& Davis, E.A. (2006). Why do some overweight children experience psychological problems? The role of weight and shape concern. International Journal Pediatric Obes., 1(4), 239-47. Recuperado de https:// www.ncbi.nlm.nih.gov/pubmed/17907331

Álvarez, C. (2018). Análisis de los niveles de actividad física de los estudiantes, el tipo de contenido de la lección y la interacción del profesor durante la clase de Educación Física de escuelas públicas. Manuscrito enviado a publicación.

Ambulo, G., González, M., \& Montenegro, R. (2018). Análisis del sobrepeso, obesidad, actividad física y autoestima de la niñez en el Área Metropolitana de la Región Educativa Panameña. Manuscrito en preparación.

Batista, M., Cubo, D. S., Honório, S., \& Martins, J. (2016). The practice of physical activity related to self-esteem and academical performance in students of basic education. Journal of Human Sport and Exercise, 11(2), 297-310. doi: https:// doi.org/10.14198/jhse.2016.112.03

Cambizaca, G., Castañeda, I., \& Sanabria, G. (2015). Sobrepeso, obesidad y diabetes mellitus 2 en adolescentes de América Latina en 2000-2010. Revista Cubana de Medicina General Integral,31(2), 217-231.
Campo T., L.A. (2014). El desarrollo del autoconcepto en niños y niñas y su relación con la interacción social en la infancia. Revista Psicogente, 17(31), 67-79. Recuperado de http:// www.scielo.org.co/scielo.php?script=sci_arttext\&pid=S012401372014000100005\&lng=en\&tlng=es.

Catalani, F., Fraire, J., Peréz, N., Mazzola, M., Martínez, A., \& Mayer, M. (2016). Prevalencia de bajo peso, sobrepeso y obesidad en adolescentes escolarizados de la provincia de La Pampa. Archivos Argentinos de Pediatría 114(2), 2-3. doi: https://doi.org/10.5546/aap.2016.154

Ceballos, C., Vizcarra, I., Acosta, L.,Reyes, C., \& Loza, M. (2012). Sobrepeso y obesidad en preescolares y escolares de una comunidad periurbana de origen otomí del Valle de Toluca, México. Revista Población y Salud en Mesoamérica, 10(1), 1-23. https://dialnet.unirioja.es/servlet/articulo?codigo=4724545

Centro de Control y Prevención de Enfermedades [CDC] (2015). Calculadora del índice de masa corporal para la población infantil y juvenil. Recuperado de https://nccd.cdc.gov/dnpabmi/ Calculator.aspx

Cigarroa, I., Sarqui, C., \& Zapata-Lamana, R. (2016). Efectos del sedentarismo y obesidad en el desarrollo psicomotor en niños y niñas: Una revisión de la actualidad latinoamericana. Universidad y Salud, 18(1), 156-169. doi: https://doi.org/10.22267/ rus.161801.27

Cofré, C., Muros, J. J., \& Zurita, F.(2015). El problema del sobrepeso y la obesidad infantil y sus interacciones con la actividad física, el sedentarismo y los hábitos nutricionales en Chile. (Tesis Doctoral). Universidad de Granada, España, 1-256. doi: https:/ /doi.org/10.4067/S0034-98872007000100009

Consejo de Ministros de Salud de Centroamérica y República. Dominicana [COMISCA]. (2013). Estrategia para la prevención del sobrepeso y la obesidad en la niñezy adolescencia de Centroamérica y República Dominicana 2014-2025. Recuperado de http://copal.org.ar/wp-content/uploads/2015/06/ 201406-comisca-estrategia_obesidad.pdf

Contreras-Valdez, J. A., Hernández-Guzmán, L., \& Freyre, M. Á. (2016). Body dissatisfaction, self-esteem, and depression in girls with obesity. Revista Mexicana de trastornos alimentarios, 7(1), 24-31. doi: https://doi.org/10.1016/ j.rmta.2016.04.001

Corvalán, C., Garmendia, M. L., Jones-Smith, J., Lutter, C. K., Miranda, J. J., Pedraza, L. S., Popkin, B. M., Ramiréz-Zea, M., Salvo, D., \& Stein, A. D. (2017). Nutrition status of children in Latin America. Obesity Reviews, 18(July), 7-18. doi: https:/ /doi.org/10.1111/obr.12571

Crocker, P.R. E., Bailey, D.A., Faulkner, R.A., Kowalski, K.C., \& McGrath, R. (1997). Measuring general levels of physical activity: Preliminary evidence for the Physical Activity Questionnaire for Older Children. Medicine and Science in Sports and Exercise, 29(10), 1344-1349. doi: https://doi.org/ 10.1097/00005768-199710000-00011

De Piero, A., Rodríguez-Rodríguez, E., González-Rodríguez, L. G., \& López-Sobaler, A. M. (2014). Sobrepeso y obesidad en un grupo de escolares españoles. Revista Chilena de Nutrición, 41(3), 264-271. doi: https://doi.org/10.4067/S071775182014000300006

Delgado, A., \& Contreras, T. (2012). La actividad física como prevención de la obesidad en niños entre 6 y 12 años en los centros educativos de la parroquia Sucre de la Ciudad de Cuenca-2012. Universidad Politécnica Salesiana: Cuenca, Ecuador. Recuperado de https://dspace.ups.edu.ec/bitstream/ 123456789/2620/14/UPS-CT002440.pdf

Delgado-Floody, P., Caamaño, F., Osorio, A., Jerez, D., Fuentes, J., Levin, E., \& Tapia, J. (2017). Imagen Corporal y Autoestima 
en niños según su estado nutricional y frecuencia de actividad física. Revista Chilena de Nutrición, 44(1), 12-18. doi: https:/ /doi.org/10.4067/S0717-75182017000100002

Delgado-Floody, P., Caamaño-Navarrete, F., Jerez-Mayorga, D., Cofré-Lizama, A., Osorio-Poblete, A., Campos-Jara, C., Guzmán-Guzmán, I., Martínez-Salazar, C., \& CarcamoOyarzun, J. (2017). Obesidad, autoestima y condición física en escolares. Revista de la Facultad de Medicina, 65(1). 43-8. doi: http://dx.doi.org/10.15446/revfacmed.v65n1.47103.

Diéguez, M. (2015). Guatemala, Décimo lugar en obesidad. Crónicas. Recuperado de http://cronica.gt/guatemala-decimo-lugar-en-obesidad/

Dubón, S., \& Aguilar, C. (2018). Análisis de sobrepeso y obesidad, niveles de actividad física y autoestima en la niñez guatemalteca. Manuscrito enviado a publicación.

Espinosa, E. (2013). Educación física restringida: Una asignatura en extinción. Recuperado de https://www.prensa.com/ re in a ld o _ w e e k s / E d u c a c i on - f i s i c a restringida_0_3613138736.html

Estado de la Región. [ERCA]. (2016). Quito Informe, Estado de la Región. Recuperado de http://www.estadonacion.or.cr/ erca2016/index.html

Evert, B. y Álvarez, C. (2018). Análisis del sobrepeso y obesidad, niveles de actividad física y autoestima de la niñez salvadoreña. Manuscrito enviado a publicación.

Fernández, X. (2014). Obesidad de escolares en Costa Rica resalta en Latinoamérica. Recuperado de http://www.nacion.com/ vivir/bienestar/Obesidad-escolares-ticos-resalta Latinoamerica_0_1401259909.html

González, G., Zurita, F., Puertas, P., Espejo, T., Chacón, R., \& Castro, M. (2017). Influencia de los factores sedentarios (dieta y videojuegos) sobre la obesidad en escolares de Educación Primaria. Reidocrea, 6(11), 120-129. Recuperado de http:// hdl.net/10481/45233

Hernández, L.A., Ferrando, J., Quílez, J., Aragonés, M., \& Terreros, J. (2010). Análisis de la actividad física en escolares de medio urbano. Madrid, España: Subdirección general de deporte y Salud. Consejo Superior de Deportes. Recuperado de http://www.csd.gob.es/csd/estaticos/documentos/ ICD55_WEB.pdf

Hernández-Sampieri, R., Fernández-Collado, C., \& Baptista, M.P. (2010). Metodología de la Investigación. México, D.F: Editorial McGraw-Hill, S.A.

Herrera-Monge, M.F., Álvarez Bogantes, C., Sánchez Ureña, B., Herrera-González, E., Villalobos Víquez, G. y Vargas Tenorio, J. (2019). Análisis de sobrepeso y obesidad, niveles de actividad física y autoestima en la niñez del II ciclo escolar del cantón central de Heredia, Costa Rica. Población y Salud en Mesoamérica, 17(1). doi: https://doi.org/10.15517/ psm.v17i1.35323

Hruby, A., \& Hu, F. B. (2015). HHS Public Access. Pharmacoeconomics., 33(7), 673-689. doi: https://doi.org/ 10.1007/s40273-014-0243-x.The

Instituto de Nutrición de Centroamérica y Panamá [INCAP]. (2015). Evolución de la Nutrición en Centroamérica y República Dominicana: Temas de la Agenda Pendiente y Problemas Emergentes. Recuperado de http://ow.ly/XDB3S

Instituto de Nutrición de Centroamérica y Panamá [INCAP].(2016) Situación de la obesidad en Centroamérica y República Dominicana. Revista A.C. Nota Técnica, 1-7. Recuperado de http:// www.incap.int/sisvan/index.php/es/cooperacion-tecnica-en-laregion/documentos-especializados/doc_view/279-nota-tecnicasituacion-de-la-obesidad-en-centro-america-y-republica-dominicana-2016
Instituto Nacional de Información de Desarrollo [INIDE]. (2013). Encuesta Nicaragüense de Demografía y Salud 2011 / 12. Informe Preliminar. Recuperado de http://www.inide.gob.ni/ endesa/Endesa11_12/HTML/endesa11/assets/common/ downloads/Informepreliminar.pdf

Janz, K. F., Lutuchy, E. M., Wenthe, P., \& Levy, S. M. (2008). Measuring activity in children and adolescents using self-report: PAQ-C and PAQ-A. Medicine \& Science in Sports \& Exercise, 40(4), 767-772. Recuperado de: https://www.ncbi.nlm.nih.gov/ pubmed/18317366

Klein, G. (2008). La calidad de la educación física y el deporte de la juventud: actualidad y retos de futuro en un mundo globalizado. Mesa Redonda presentada en el V Congreso Internacional de Educación Física, Barcelona, España.

Lacayo, M., Check, E., Vega, N. F., \& Córdova,A. (2018). Análisis de sobrepeso y obesidad, niveles de actividad física y autoestima en la niñez de Tegucigalpa, Honduras. Manuscrito en preparación.

Liria, R. (2012). Consecuencias de la obesidad en el niño y el adolescente: un problema que requiere atención. Revista Peruana de Medicina Experimental y Salud Pública, 29(3), 357-60. Recuperado de https://www.scielosp.org/pdf/rpmesp/ 2012.v29n3/357-360/es

Lizardo, A.E., \& Díaz, A. (2011). Sobrepeso y obesidad infantil. Revista Médica de Honduras, 79(4), 208-213. Recuperado de www.bvs.hn/RMH/pdf/2011/pdf/Vol79-4-2011-9.pdf

Macias, A. I., Gordillo L. G., \& Camacho, E. J. (2012). Hábitos alimentarios de niños en edad escolar y el papel de la educación para la salud. Revista Chilena de Nutrición, 39(3), 40-43. doi: https://dx.doi.org/10.4067/S0717-75182012000300006

Marcó, M., \& de Gracia, M. (2000). Evaluación de la Imagen Corporal en preadolescentes. Girona, España: Universitat de Girona.

Martínez, C. P., Cuberos, R. C., Sánchez, M. C., Garcés, T. E., Ortega, F. Z., \& Cortés, A. J. P. (2017). Diferencias de género en relación con el Índice de Masa Corporal, calidad de la dieta y actividades sedentarias en niños de 10 a 12 años. Retos: nuevas tendencias en educación física, deporte y recreación, (31), 176-180. Recuperado de https://dialnet.unirioja.es/servlet/ articulo?codigo $=5841367$

Martínez-Gómez, D., Martínez-de-Haro, V., Pozo, T., Welk, G. J., Villagra, A., Calle, M. E., Marcos, A., \& Veiga, O. L. (2009). Fiabilidad y validez del cuestionario de actividad física PAQ-A en adolescentes españoles. Revista Española de Salud Pública, 83(3), 427-439. doi: https://doi.org/10.1590/S113557272009000300008

Mazidi, M., Banach, M., Pascal-Kengne, A., \& Lipid and Blood Pressure Meta-analysis Collaboration Group. (2018). Prevalence of childhood and adolescent overweight and obesity in Asian countries: a systematic review and meta-analysis. Arch Med Sci, 14(6), 1185-1203. doi: https://doi.org/10.5114/ aoms.2018.79001

Medina, C., Yuja, N., \& Lanza, O. (2016). Prevalencia de Sobrepeso y Obesidad en niños de 6 a 12 años de tres escuelas de Honduras. Archivos de Medicina, 12(3), 1-7. doi: https://doi.org/ $10.3823 / 1312$

Meyers, L.S., Gamst, G., \& Guarino, A. J. (2013). Applied multivariate research: design and interpretation (2 ed.). California: Editorial Sage.

Ministerio de Economía y Finanzas de Panamá [MEF]. (2008). Panamá - Encuesta de Niveles de Vida 2008. Recuperado de http://microdata.worldbank.org/index.php/catalog/70

Ministerio de Salud de El Salvador [MINSAL]. (2016). MINSAL realiza taller de prevención de la obesidad en la niñez. Recu- 
perado de http://www.salud.gob.sv/07-09-2016-minsalrealiza-taller-de-prevencion-de-la-obesidad-en-la-ninez/

Ministerio de Salud de la Nación. (2013). Sobrepeso y obesidad en niños y adolescentes. Orientaciones para su prevención, diagnóstico y tratamiento en Atención Primaria de la Salud. Recuperado de http:// www.msal.gob.ar/images/stories/bes/graficos/ 0000000377cnt-sobrepeso-y-obesidad-en-ninios.pdf

Ministerio de Salud y Ministerio de Educación Pública. (2016a). Censo Escolar Peso/Talla 2016. Resultados., 1-51. Recuperado de http://www.mep.go.cr/sites/default/ files/page/adjuntos/resultados-censo-escolar-peso-talla2016.pdf

Ministerio de Salud y Ministerio de Educación Pública. (2016b). Informe Ejecutivo. Censo Escolar Peso/Talla Costa Rica, 2016. 1-42. Recuperado de http:// www.mep.go.cr/sites/default/files/page/adjuntos/informe-ejecutivo-censo-escolar-peso-cortofinal.pdf

Miranda-Ríos, L., Vásquez-Garibay, E. M., Romero-Velarde, E., Nuño-Cosío, M. E., Campos-Barrera, L., Caro-Sabido, E. A., \& Ramírez-Díaz, J. (2017). Factores asociados a la actividad física y el índice de masa corporal en escolares de Arandas, Jalisco, México. Revista Médica del Instituto Mexicano del Seguro, 55(4), 472-80. Recuperado de http://www.redalyc.org/articulo.oa?id=457751260011

Muñoz, G. (2017). Una minoría recibe Educación Física. Periódico Prensa Libre.Guatemala. Recuperado de http:/ /www.prensalibre.com/guatemala/comunitario/unaminoria-recibe-educacion-fisica

Naranjo M. (2007). Autoestima: un factor relevante en la vida de la persona y tema esencial del proceso educativo. Revista Electrónica publicada por el Instituto de Investigación en Educación, 77(3), 1-27. doi: https:// doi.org/10.15517/aie.v17i3.29066

Nestle, M. (2007). Dietética elemental. Revista investigación y ciencia, 374, 20-29.

O’ Donoghue, P. (2012). Statistics for Sport and Exercise Studies. An Introduction. New York: Taylor and Francis.

Organización Mundial de la Salud [OMS] (2010). Recomendaciones Mundiales sobre Actividad Física para la Salud. Geneva: WHO Library Cataloguing-in-Publication, (Completo), 1-58. doi: https://doi.org/9789243599977

Organización Mundial de la Salud y Organización Panamericana de la Salud. (OMS y OPS, 2014). Plan de acción sobre la prevención de la obesidad en niños y adolescentes 2014-2019. Sesión No. 154, Comité Ejecutivo.

Organización Mundial de la Salud. [OMS]. (2000). Obesidad: prevención y manejo de la epidemia global. Geneva, Suiza. OMS Reporte Técnico Serie 894.

Organización Mundial de la Salud. [OMS]. (2018). Inactividad física: un problema de salud pública mundial. Recuperado de https://www.who.int/dietphysicalactivity/ factsheet_inactivity/es/

Raimann, X., \& Verdugo, F. (2012). Actividad física en la prevención y tratamiento de la obesidad infantil. Revista Médica Clínica Las Condes, 23(3), 218-225. doi: https:/ /doi.org/10.1016/S0716-8640(12)70304-8

Reyes, R., Otero, E., Pastrán, R., Herrera-Monge, M., \& Álvarez, C. (2018). Análisis del sobrepeso, obesidad, niveles de actividad física y autoestima de la niñez de León, Nicaragua. MHSalud: Revista en Ciencias del Movimiento Humano y Salud, 16(1), 1-15. doi: https://doi.org/ 10.15359/mhs.16-1.4
Sánchez, J., \& Ruiz, A. (2015). Relationship between selfesteem and body image in children with obesity. Revista Mexicana de Trastornos Alimentarios, 6(1), 38-44. doi: https://doi.org/10.1016/j.rmta.2015.05.006

Savino, P. (2011). Obesidad y enfermedades no transmisibles relacionadas con la nutrición. Revista Colombiana de Cirugía, 26, 180-195. Recuperado de http:// www.scielo.org.co/pdf/rcci/v26n3/v26n3a5.pdf

Shamah, T. (2016). El sobrepeso y la obesidad: ¿ son una situación irremediable? Boletín Médico Del Hospital Infantil de México, 73(2), 65-66. doi: https://doi.org/ 10.1016/j.bmhimx.2016.02.002

Solano L., Baron M., \& Del Real, S. (2005). Situación nutricional de preescolares, escolares, y adolescentes de Valencia, Carabobo, Venezuela. Anales Venezolanos de Nutrición, 18(1), 72-76. Recuperado de http:// w w w . S c i e l o .0 o scielo.php? script =sci_arttext \&pid=S079807522005000100014

Taberno, C., Serrano, A., \& Mérida, R. (2017). Estudio comparativo de la autoestima en escolares de diferente nivel socioeconómico Carmen. Psicología Educativa, 23, 917. doi: https://doi.org/http://dx.doi.org/10.1016/ j.pse2017.02.001

Torres, G., Carpio, E., Lara, A. J., \& Zagalaz, M. L. (2014). Niveles de condición física de escolares de educación primaria en relación a su nivel de actividad física y al género. Retos: Nuevas Tendencias En Educación Física, Deporte y Recreación, 2041(25), 17-22. Recuperado de https://dialnet.unirioja.es/servlet/ articulo?codigo $=4554671$ \&info=resumen\&idioma=ENG

Trejo, P., Jasso, S., Mollinedo, F., \& Lugo, L. (2012). Relación entre actividad física y obesidad en escolares. Revista Cubana de Medicina General Integral, 28(1), 34-41. Recuperado de http://scielo.sld.cu/ scielo.php? script=sci_arttext \&pid=S086421252012000100005

Vásquez-Bonilla, A., Zelaya-Paz, C., \& García-Aguilar, J. (2018). Análisis de sobrepeso y obesidad, niveles de actividad física y autoestima de la niñez de San Pedro Sula, Honduras. Manuscrito en preparación.

Villagrán, S., Rodríguez-Martín, A., Novalbos, J. P., Martínez, J. M., \& Lechuga Campoy Hábitos y estilos de vida modificables en niños con sobrepeso y obesidad. Revista Nutrición Hospitalaria, 25(5), 823-831. Recuperado de http://scielo.isciii.es/scielo.php?pid=S0212$16112010000500022 \&$ script=sci_arttext\&tlng=en

Zamani, S. H., Fathirezaie, Z., Brand, S., Pühse, U., HolsboerTrachsler, E., Gerber, M., \& Talepasand, S. (2016). Physical activity and self-esteem: Testing direct and indirect relationships associated with psychological and physical mechanisms. Neuropsychiatric Disease and Treatment, 12, 2617-2625. doi: https://doi.org/10.2147/NDT.S116811

Zanatta, L. D. P., Fuentes, N. I. G. A. L., van Barneveld, H. O., Medina, J. L. V., \& Escobar, S.G. (2014). Factores psicológicos asociados con el rendimiento escolar en estudiantes de educación básica. Revista Intercontinental de Psicología y Educación, 16(2), 131-149. Recuperado de http://www.redalyc.org/articulo.oa?id=80231541008 